\title{
Ingredients, Functionality, and Safety of the Japanese Traditional Sweet Drink Amazake
}

\author{
Atsushi Kurahashi $\mathbb{D}$ \\ Hakkaisan Brewery Co., Ltd., 1051 Nagamori, Minamiuonuma 949-7112, Niigata, Japan; \\ a.kurahashi@hakkaisan.jp
}

check for updates

Citation: Kurahashi, A. Ingredients, Functionality, and Safety of the Japanese Traditional Sweet Drink Amazake. J. Fungi 2021, 7, 469. https://doi.org/10.3390/jof7060469

Academic Editors: Katsuhiko Kitamoto and Yujiro Higuchi

Received: 24 May 2021

Accepted: 7 June 2021

Published: 10 June 2021

Publisher's Note: MDPI stays neutral with regard to jurisdictional claims in published maps and institutional affiliations.

Copyright: (c) 2021 by the author Licensee MDPI, Basel, Switzerland. This article is an open access article distributed under the terms and conditions of the Creative Commons Attribution (CC BY) license (https:// creativecommons.org/licenses/by/ $4.0 /$ )
Abstract: The sweet drink amazake is a fermented food made from Aspergillus oryzae and related koji molds in Japan. There are two types of drinks called amazake, one made from koji (koji amazake) and the other made from sake lees, a by-product of sake (sakekasu amazake). The sweetness of koji amazake is from glucose, derived from starch broken down by A. oryzae amylase. The other, sakekasu amazake, depends on added sugar. The main components are glucose and sucrose, but they also contain more than 300 other ingredients. Koji amazake contains oligosaccharides and ergothioneine, and sakekasu amazake has a resistant protein and $\alpha$-ethyl glucoside, which are characteristic ingredients of each amazake. However, there are also common ingredients such as glycosylceramide. Functionality is known to include anti-fatigue, bowel movement, skin barrier, and other effects on human health. In particular, the bowel movement-improving effects have been well studied for both amazakes. These functions result from ingesting approximately $100 \mathrm{~mL}$ per day, but human clinical trials have clarified that this amount has no effect on blood glucose levels and weight gain. In the future, the identification of substances associated with each function is required.

Keywords: amazake; Aspergillus oryzae; koji; sake lee; glucose; anti-fatigue; bowel movement; skin barrier

\section{Introduction}

There are a wide variety of traditional fermented foods made from Aspergillus oryzae and related koji molds in Japan, such as sake, shochu (Japanese traditional hard liquor), mirin (sweet sake), rice vinegar, soy sauce, miso, salt koji, and some Japanese pickles. Koji amazake is the simplest fermented product. Koji is a grain, such as rice, wheat, and soybeans, fermented with $A$. oryzae and with related koji molds grown on it. There are two types of drinks called amazake, one made from rice-koji and the other made from sake lees, which is a by-product of sake. Both amazakes are closely related to sake brewing (Figure 1). Sake brewing begins by polishing brown rice into white rice. The polished rice is steamed, and the spores of $A$. oryzae (tane-koji) are then inoculated on the steamed rice. Rice-koji is produced based on the growth of $A$. oryzae from the surface layer to the rice (rice-koji making process). Sake yeast is cultivated using steamed rice and rice-koji (Shubo making process). In addition, steamed rice, rice-koji, and Shubo are combined (moromi) for alcoholic fermentation. After alcoholic fermentation, the mash is pressed and separated into liquid and solid sake lees.

Koji amazake is a non-alcoholic, white-colored, sweet, traditional Japanese drink (Figure 2). Moreover, it is divided into two types depending on the raw material used. One type is made only from rice-koji and water, whereas the other type is made with additional rice. Therefore, koji amazake includes rice and A. oryzae products. The koji amazake production process is as follows: the rice-koji is mixed with water and placed in a tank set at 50-60 ${ }^{\circ} \mathrm{C}$, where the amylase secreted by $A$. oryzae breaks down rice starch into glucose. It is a unique beverage for which the main component is glucose, as compared to other sweet beverages such as fruit juice, for which the main sugars are sucrose and fructose. Tape ketan in Indonesia and Loog-pang in Thailand, which resemble koji amazake, are foods 
for which grains are saccharified by the amylase produced by filamentous fungi and yeasts, such as Amylomyces rouxii and Saccharomycopsis fibuligera [1,2]. These are sweet and sour foods that include alcohol, due to alcoholic fermentation and lactic acid fermentation by mixed microorganisms, and are different from koji amazake. The other amazake, sakekasu amazake, is a sweet drink made from dissolved sake lees in water and the addition of sugar. Unlike koji amazake, it contains sake yeast, its metabolites, and a small amount of alcohol produced by alcoholic fermentation. Other amazakes, comprising a mixture of sake lees and rice-koji, are also called sakekasu amazake. In this review, some amazakes containing both rice-koji and sake lees, but depending on which amount is larger, are called koji amazake or sakekasu amazake.

\section{O Sake brewing process}

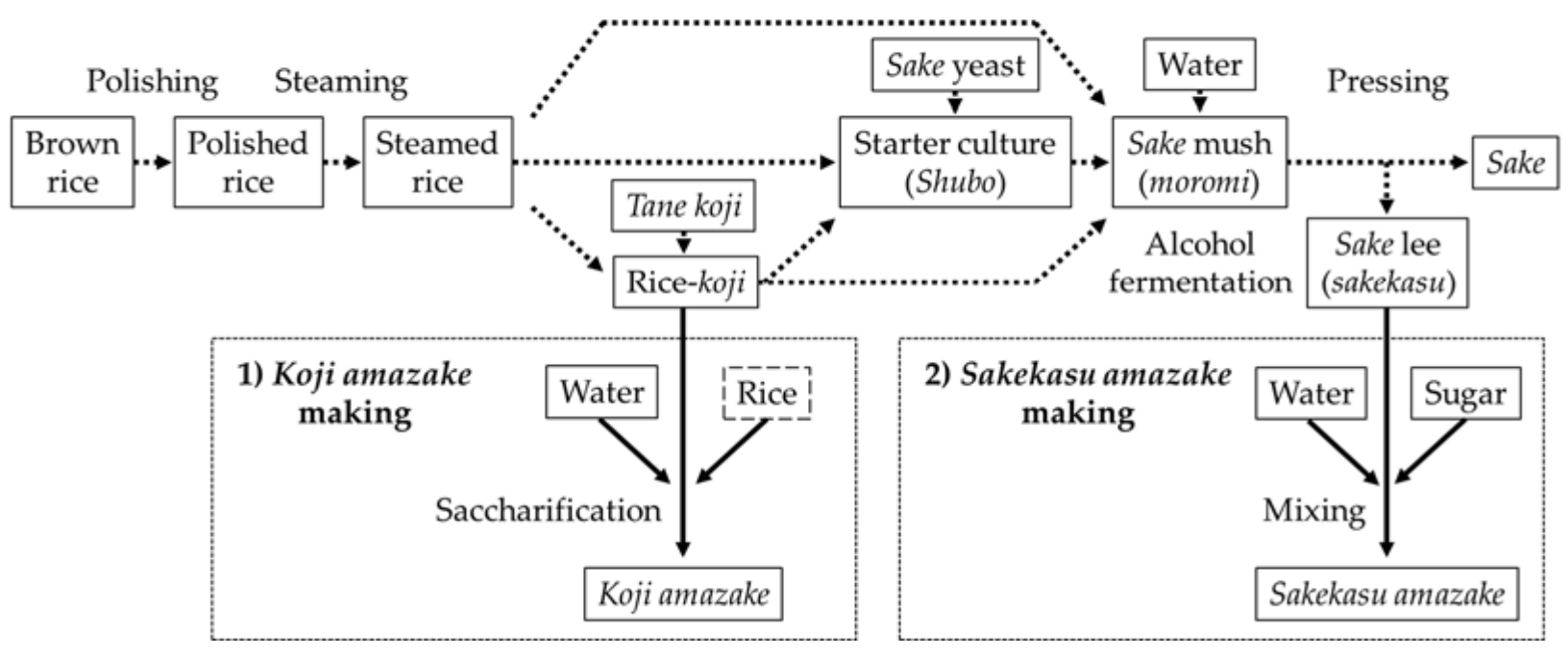

Figure 1. Relationship between sake brewing and the amazake production process.

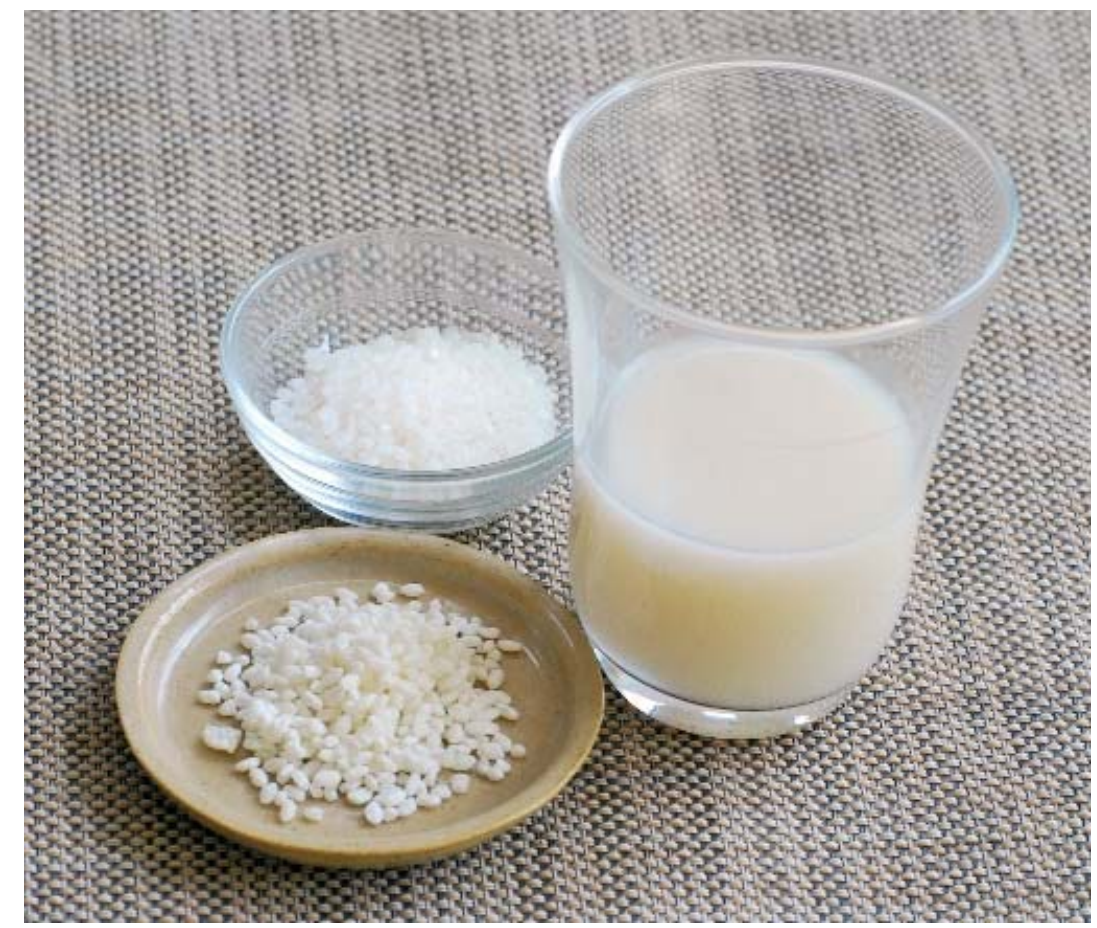

Figure 2. Rice, rice-koji and koji amazake. 
In particular, koji amazake has been consumed for a long time, as it appeared in the Chronicles of Japan (Nihon shoki) compiled in 720, the second oldest book of classical Japanese history; however, in the middle of the Edo period (around 1700-1750), it was made by the same method as it is today and sold by peddlers [3] (Figure 3). In Japan, people prefer koji amazake as a nutritional supplement to prevent heat fatigue in the summer, as in the early modern period in Japan. It is thought that sakekasu amazake started to be consumed at the end of the Taisho period as a substitute for koji amazake. Another drink, in which sake lees is dissolved in hot water (kasuyuzake), has also been consumed for a long time as a substitute for sake [4]. Koji amazake and sakekasu amazake, including kasuyuzake, have been consumed for a long time. Many studies have been conducted on the ingredients and functionality of rice-koji and sake lees, which are the raw materials used for amazake. Nevertheless, there are few research reports on both amazake and other fermented foods using koji. Recently, an amazake boom occurred in Japan in 2015, and its market size grew from JPY 11.9 billion in 2009 to JPY 16.7 billion in 2015 and JPY 24.6 billion in 2017 [5]. In addition, research reports, including those on safety, have increased. This review introduces information that is currently known regarding this issue.

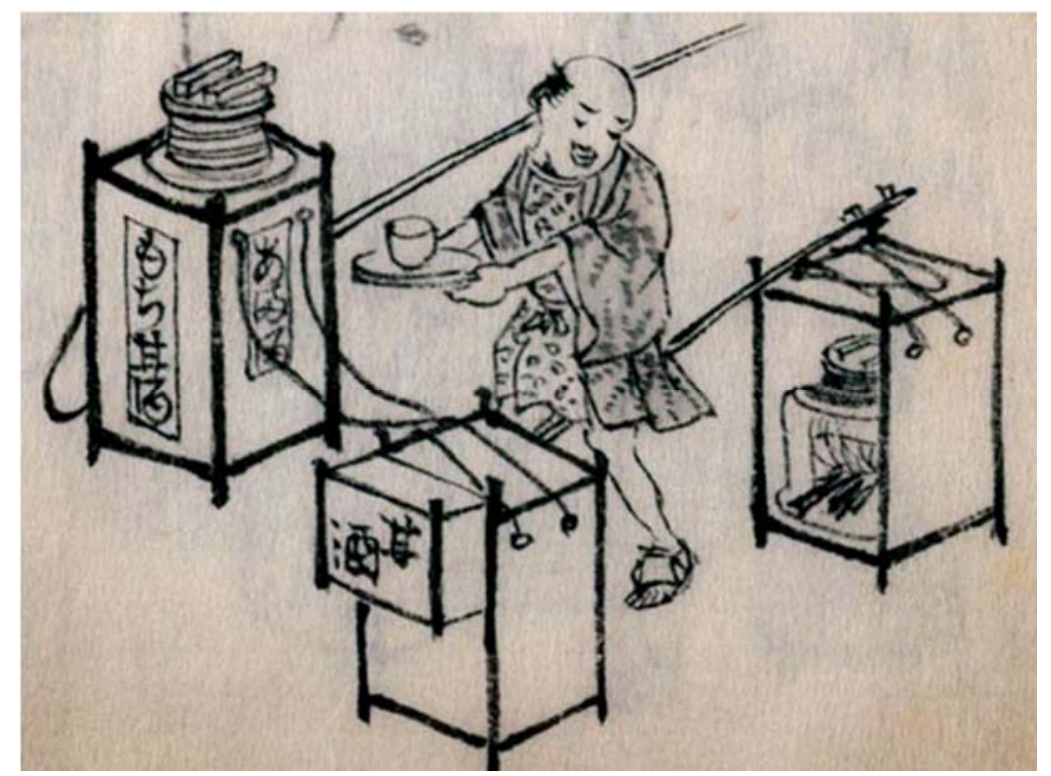

Figure 3. Koji amazake peddler in the Edo period [3].

\section{Ingredients}

Examples of the general nutritional components of koji amazake and sakekasu amazake are shown in Table 1. Carbohydrates are the most abundant nutritional component of both amazakes, but their contents are different. Most of the carbohydrates in koji amazake are glucose derived from rice starch, which is broken down by $\alpha$-amylase and glucoamylase secreted by $A$. oryzae, as described previously herein. Furthermore, various oligosaccharides, mainly glucooligosaccharides, are produced by the transglycosylation activity of $\alpha$-glucosidase, including trehalose (Glc $(\alpha 1-1) G l c)$, kojibiose (Glc $(\alpha 1-2) G l c)$, nigerose $(\mathrm{Glc}(\alpha 1-3) \mathrm{Glc})$, maltose (Glc $(\alpha 1-4) \mathrm{Glc})$, isomaltose (Glc $(\alpha 1-6) \mathrm{Glc})$, sophorose (Glc $(\beta 1-2) \mathrm{Glc})$, and gentiobiose (Glc $(\beta 1-6)$ Glc) (disaccharides), as well as maltotriose $(\mathrm{Glc}(\alpha 1-4) \mathrm{Glc}(\alpha 1-$ $4) \mathrm{Glc})$, isomaltotriose (Glc $(\alpha 1-6) \mathrm{Glc}(\alpha 1-6) \mathrm{Glc})$, panose $(\mathrm{Glc}(\alpha 1-6) \mathrm{Glc}(\alpha 1-4) \mathrm{Glc})$, and raffinose $(\mathrm{Gal}(\alpha 1-6) \mathrm{Glc}(\beta 1-2) \mathrm{Fru})$ (trisaccharides) [6-10]. The oligosaccharide present in the largest amount is isomaltose, which accounts for approximately 2-3\% [6]. The main sugar in sakekasu amazake is added sucrose.

In addition to the main sugar, koji amazake contains more than 300 compounds [9]. Twenty amino acids are produced by the degradation of rice proteins by the protease of $A$. oryzae $[9,11]$. Other amino acids include $\gamma$-aminobutyric acid and ergothioneine (EGT) [9]. The number of amino acids depends on the amount of rice koji and steamed rice, but the 
rice polishing rate also has an effect on amino acid content because proteins are unevenly distributed on the surface layer of rice. The vitamin B complex includes thiamine (B1), riboflavin (B2), nicotinic acid (B3), pantothenic acid (B5), pyridoxine (B6), and biotin (B7) in koji amazake [9-15]. The $\mathrm{B}$ vitamins contained in rice bran are removed by polishing; these vitamins are also produced by $A$. oryzae during the rice-koji making process. Lipids in koji amazake include palmitic acid, oleic acid, and linoleic acid [9]. Glycosylceramide is included as another lipid component that has been reported in several structures derived from the cell membrane of $A$. oryzae as follows: $N-2^{\prime}$-hydroxyoctadecanoyl-l-O- $\beta$-D-glucopyranosyl9-methyl-4,8-sphingadienine and $\mathrm{N}-2^{\prime}$-hydroxyoctadecanoyl-l-O- $\beta$-D-galactopyranosyl-9methyl-4,8-sphingadienine [16-18]. In addition, although those involved are unknown, koji amazake has been reported to contain substances with antioxidant activity [19]. The characteristic ingredients of sakekasu amazake are resistant proteins [20] and $\alpha$-ethyl-D-glucoside $(\alpha-E G)$ [21] produced by the sake brewing process. Resistant proteins are indigestible proteins resistant to proteases in rice-koji and have a dietary fiber-like effect. $\alpha$-EG is produced by the transfer of ethanol to glucose, mediated by $\alpha$-glucosidase in rice-koji.

Table 1. Nutritional components of koji amazake, sakekasu amazake, and rice syrup as a placebo (per $100 \mathrm{~g}$ ).

\begin{tabular}{lccc}
\hline Nutritional Components & Koji amazake & Sakekasu amazake & Rice Syrup \\
\hline Energy $(\mathrm{kcal})$ & 105 & 102 & 106 \\
Carbohydrate(CHO) $(\mathrm{g})$ & 24.8 & 23.1 & 23.1 \\
Dietary fiber $(\mathrm{g})$ & 0.3 & 0.5 & 0.5 \\
Available CHO $(\mathrm{g})$ & 24.5 & 22.6 & 24.8 \\
Glucose $(\mathrm{g})$ & 23.2 & 2.1 & 23.3 \\
Sucrose $(\mathrm{g})$ & $\mathrm{ND}$ & 13.8 & $\mathrm{ND}$ \\
Protein $(\mathrm{g})$ & 1.3 & 1.4 & 1.4 \\
Fat $(\mathrm{g})$ & 0.1 & 0.5 & 0.2 \\
\hline
\end{tabular}

\section{Functionality}

Koji amazake has a history of being consumed as a nutritional supplement to prevent heat fatigue, and its effects on improving bowel movements and the skin barrier are empirically known. While scientific verification is underway, other functional properties have also become clear in the verification process.

\subsection{Anti-Fatigue Effect}

Nagao and Sata investigated an improvement in the quality of life (QOL) in four patients (two males and two females) with viral liver cirrhosis after ingesting a late evening snack of koji amazake as a pilot study [12]. Serum biochemical parameters and the visual analogue scale were examined at $0,4,8$, and 12 weeks. Koji amazake intake improved QOL based on all investigated terms, such as fatigue. The authors concluded that $B$ vitamins and amino acids, especially branched chain amino acids (BCAAs), contained in koji amazake, might be functional substances. Koji amazake has also been reported to have remarkable physical fatigue recovery effects on pain in male and female long-distance runners [22]. In that study, it was inferred that the functional substances might be BCAAs. It has further been reported that BCAAs reduce muscle pain and fatigue caused by exercise [23], but the amount of BCAAs contained in koji amazake is lower [6]; therefore, this effect is considered to be due to other ingredients or a combined effect.

\subsection{Bowel Movement Improvement}

The effect of improving bowel movements mediated by koji amazake intake has been reported in two studies. Sumiyoshi and Nakao studied the effect of koji amazake on constipation by comparing a koji amazake intake group (11 females), administered $150 \mathrm{~mL} /$ day for 2 weeks, with a non-drinking group (seven females) [24]. The koji amazake intake group showed a significant improvement in the frequency of bowl movements and constipation. 
Further, Sakurai et al. reported the effects of koji amazake on defecation status in healthy volunteers with relatively low stool frequency [25]. The koji amazake intake group (five males, nine females) had an improved defecation frequency compared to that before intake. However, there were no significant changes in the intestinal microbiota.

The effect of improving bowel movements has also been reported for sakekasu amazake. The number of defecations increased significantly in 12 volunteers (five males and seven females) who continued to drink sakekasu amazake for 3 weeks every day [20]. Mori et al. reported the effect of the intake of sakekasu amazake containing both sake lees and rice-koji on improving bowel movements [26]. The defecation frequency was compared between the sakekasu amazake intake group (21 volunteers of unknown sex) who drank $190 \mathrm{~g}$ of amazake for 30 days and the placebo group (17 volunteers) who drank $190 \mathrm{~g}$ of hot water. The amazake intake group showed an improved number of bowel movements, number of stools, state of the stool, and sensation after defecation per bowel movement compared to those in the placebo group. Moreover, Mori et al. reported the effect of koji amazake and sakekasu amazake intake on the human intestinal microbiota in a randomized placebo-controlled crossover comparison study [27]. The koji amazake intake group (13 females) and sakekasu amazake intake group (12 females) ingested $125 \mathrm{~mL}$ of the koji amazake (or sakekasu amazake) or placebo for 30 days, whereas other beverages were ingested after a 14-day washout period. The koji amazake intake group showed no significant increase in Bifidobacterium, but the sakekasu amazake intake group showed a significant increase. In contrast, Maruki-Uchida et al. reported that the microbiota was unchanged with the intake of sakekasu amazake, as compared to that with placebo [28]. This study was a randomized, placebo-controlled, double-blind trial involving healthy female volunteers. The sakekasu amazake intake group (nine females) and placebo intake group (eight females) ingested $100 \mathrm{~mL}$ of the sakekasu amazake or placebo twice per day for 4 weeks.

In animal studies, the effects of sakekasu amazake and glycosylceramides of A. oryzae on the intestinal microbiota have been reported. Kawakami et al. reported that the intake of sakekasu amazake increased mucin levels and altered the intestinal microbiota in mice [29]. The function of the glycosylceramides contained in A. oryzae as a prebiotic for Blautia coccoides in mice has also been reported [30]. These studies showed that amazake improves bowel movement; however, further studies are required on the underlying mechanism and functional substances.

\subsection{Skin Barrier Function}

Ueda et al. reported the effect of koji amazake intake on skin barrier function [31]. This study was conducted based on a randomized, double-blind, placebo-controlled, parallelgroup design with healthy adult female volunteers with moderately dry skin conditions. The koji amazake intake group (32 females) and placebo intake group (32 females) ingested $125 \mathrm{~mL}$ of koji amazake or placebo for 8 weeks. Trans-epidermal water loss of the left cheek in the koji amazake intake group in the 4th week of observation was significantly improved compared to that in the placebo intake group. The authors inferred that glucosylceramide (GlcCer) and $\mathrm{N}$-acetylglucosamine (GlcNAc) were the functional substances. The effects of GlcCer on skin barrier function have been reported for rice [32], corn [33], konjac [34], and beet [35]. The structure of GlcCer in koji amazake is similar to that of GlcCers and might have the same effect. GlcNAc, a cell wall component of A. oryzae GlcNAc has been reported to improve dry skin with intake of $500 \mathrm{mg} /$ day for 4 and 8 weeks [36]. However, since the amount of GlcNAc contained in koji amazake is less than that amount [37], other substances might be involved. EGT contained in koji amazake is one such candidate as a functional ingredient affecting skin barrier function. As such, EGT has been reported to improve UVA-induced skin aging [38].

It has been reported that the skin barrier is improved by sakekasu amazake, as well as by koji amazake. Watanabe reported that the intake of sakekasu amazake improved skin texture [20]. The improvement of the intestinal environment by the resistant protein contained in sakekasu amazake and the effect of free amino acids have also been discussed. 
Maruki-Uchida et al. also reported that the intake of sakekasu amazake improved skin properties based on questionnaires [28]. In particular, skin color measurements revealed increased brightness ( $\mathrm{L}^{*}$ and $\mathrm{R} 520+\mathrm{R} 650$ values) under the eye skin. This report also discussed the possibility that free amino acids, such as BCAA and arginine, could improve skin color through blood flow. In mouse and cell studies reported on $\alpha$-EG $[39,40]$, this characteristic component of sakekasu amazake has been reported. However, verification of the effect of $\alpha$-EG on the human skin barrier requires further in vivo studies.

\subsection{Other Functionality}

The intake of koji amazake was reportedly associated with the possibility of relieving arthralgia based on an anti-aging QOL common inquiry test [37]. This study was a randomized, double-blind, placebo-controlled, parallel-group trial. The koji amazake intake group (14 males and 8 females) and placebo (rice syrup) intake group (14 males and 8 females) ingested $118 \mathrm{~g}$ of the koji amazake or placebo daily for 12 weeks. Rice syrup was prepared using rice, which is the raw material of rice-koji, and a saccharifying enzyme that has the same composition as koji amazake (Table 1). GlcNAc has been reported to improve cartilage metabolism via the intake of 500 and $1000 \mathrm{mg} /$ day [41]. However, GlcNAc in koji amazake has a lower content than these effect sizes, as mentioned in the section on skin barrier function. Koji amazake has also been reported to contain ingredients that suppress postprandial blood glucose and insulin elevation [42]. That study was a randomized, single-blind, crossover comparative trial of koji amazake and placebo (rice syrup) ingested at $118 \mathrm{~g}$ based on healthy adult volunteers (10 males and 8 females). To verify this effect, glucose absorption was significantly inhibited by koji amazake based on an experiment using everted rat inverted intestinal sacs. Some sugars have also been reported to have an inhibitory effect on glucose absorption [43,44], and oligosaccharides and/or A. oryzae cells contained in koji amazake might be involved. In animal studies, GlcCer has been reported to significantly increase cholesterol metabolism through the expression of CYP7A1 and ABCG8 in obese mice [45].

Watanabe reported a decrease in LDL cholesterol and an increase in HDL cholesterol, in addition to improvements in bowel movements and skin barrier function, with the intake of sakekasu amazake [20]. In an animal study, Oura et al. reported that sakekasu amazake could prevent lifestyle-related diseases in mice [46]. In an obesity control study, weight gain and serum triglyceride levels were significantly suppressed in a high-fat diet including sakekasu amazake-fed group as compared to those in the control high-fat diet-fed group. In a test for the suppression of blood pressure increase, a sakekasu amazake-fed group maintained low blood pressure compared to that with the control diet intake group based on a hypertension model. Furthermore, an amnesia suppression test was conducted using mice in which scopolamine was administered to induce this condition. Although scopolamine administration diminished the learning memory of the platform-type water maze, the sakekasu amazake-fed group had a shortened time to reach the platform. These reports referred to dietary fiber, peptides, and resistant proteins as functional substances previously herein, koji amazake and sakekasu amazake have high glucose and sucrose contents, respectively. In both adults and children, the World Health Organization recommends reducing free sugar intake to less than $10 \%$ of total energy intake [47] and drinking approximately $100 \mathrm{~mL}$ of amazake daily.

\section{Safety}

As mentioned, amazake does not exceed this guideline. However, continuous drinking might lead to the development of diabetes and obesity. To address this concern, Kurahashi et al. confirmed its safety in an excessive intake test [48] and a long-term intake test of koji amazake [37]. An excessive intake test was conducted as an open trial with 24 volunteers (19 males and 5 females) who were hyperglycemic (blood sugar 100-109 mg/dL) or had borderline diabetes (110-125 mg/dL). Volunteers ingested three times more ( $379 \mathrm{kal}, 88.5 \mathrm{~g}$ of carbohydrate in total) than $118 \mathrm{~g}$ per day of koji amazake for 4 weeks. No adverse 
events were observed, blood chemistry was within the reference range, and body weight remained unchanged during the test period. A long-term intake test was conducted with 22 volunteers (14 males and 8 females) based on the same condition as the excessive intake test. The volunteers ingested $118 \mathrm{~g}$ per day for 12 weeks. There were no adverse events and no effect on body weight compared to that before koji amazake intake. As a result of a study of postprandial responses in blood glucose and insulin after the ingestion of koji amazake, the average maximum blood glucose level was $133.5 \pm 20 \mathrm{mg} / \mathrm{dL}$ and the blood glucose level increased to $46 \mathrm{mg} / \mathrm{dL}$ [42]. This value was lower than that for the same amount of cooked rice $[49,50]$. These results confirmed a safe daily intake of $118 \mathrm{~g}$ of koji amazake.

Ui reported bacterial food poisoning caused by koji amazake, mainly through staphylococci [51,52]. Koji amazake is saccharified at $50-60{ }^{\circ} \mathrm{C}$, as mentioned in the introduction. Food-poisoning bacteria do not grow in this temperature range, and it has been reported that Salmonella and Staphylococcus are sterilized above $55^{\circ} \mathrm{C}$ [53]. However, as food poisoning bacteria can grow at approximately $40{ }^{\circ} \mathrm{C}$, the saccharification temperature of koji amazake should be kept at $50-60^{\circ} \mathrm{C}$. Furthermore, after production, low-temperature $\left(>10^{\circ} \mathrm{C}\right)$ storage is required to suppress the growth of food-poisoning bacteria. Although most manufacturers sterilize amazake before distribution, it is important to note that after opening it, it should be maintained at low-temperature storage.

\section{Conclusions}

There are two types of amazake, koji amazake made from rice-koji and sakekasu amazake made from sake lees, both of which are sweet drinks that Japanese people have enjoyed since ancient times. It is not only a sweet drink but also attractive because it has a lot of functionality (Figure 4). These two types of amazake, although the ingredients and manufacturing methods are different, are interesting because they have similar effects. It is expected that more functionalities will be revealed by advancing research on amazakes. In addition, the functional substances must be identified. In the future, we hope that amazake will be consumed in many countries, including Japan, and contribute to human health.

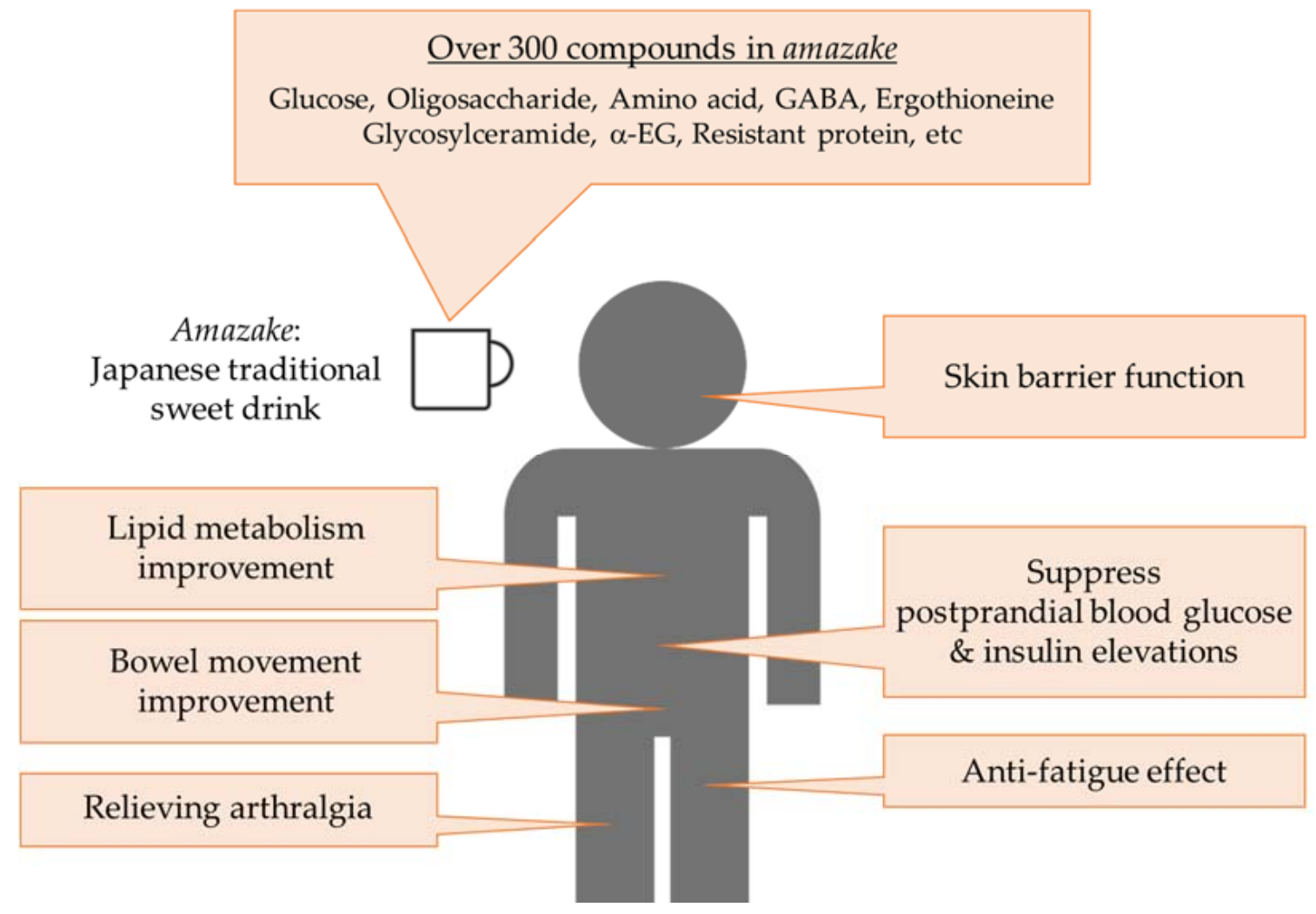

Figure 4. Summary figure describing the ingredients and functionality in amazake for human health. 
Funding: This research received no external funding.

Institutional Review Board Statement: Not applicable.

Informed Consent Statement: Not applicable.

Data Availability Statement: Not applicable.

Conflicts of Interest: This study was funded by Hakkaisan Brewery Co., Ltd., a koji amazake manufacturing company. AK is the managing director for production, and research and development.

\section{References}

1. Cronk, T.C.; Steinkraus, K.H.; Hackler, L.R.; Mattick, L.R. Indonesian Tapé Ketan fermentation. Appl. Environ. Microbiol. 1977, 33, 1067-1073. [CrossRef] [PubMed]

2. Daroonpunt, R.; Tanasupawat, S.; Keeratipibul, S. Characterization and amylolytic activity of yeast and mold strains from Thai sweet rice. Mal. J. Microbiol. 2016, 12, 121-131. [CrossRef]

3. Morisadamanko. National Diet Library Digital Collections. Available online: https://dl.ndl.go.jp/info:ndljp/pid/2592395/23 (accessed on 11 May 2021).

4. Bingu-mondoka. National Diet Library Digital Collections. Available online: https://dl.ndl.go.jp/info:ndljp/pid/874586/36 (accessed on 17 May 2021).

5. Fuji Keizai Co. Ltd. Foodstuff Marketing Handbook 2021; Fuji Keizai: Tokyo, Japan, 2020; Volume 4, pp. 342-345.

6. Kurahashi, A.; Oguro, Y. Ingredients in koji Amazake. J. Brew. Soc. Jpn. 2017, 112, 668-674.

7. Aso, K.; Watanabe, T.; Hanno, Y.; Watanabe, K. On the sugar composition of amasake (a sweet sugary liquor made from rice). Tohoku J. Agric. Res. 1962, 13, 257-264.

8. Shikata, H.; Tsukiyama, R.; Ishigami, Y.; Nishiyama, T.; Moriguchi, S. Components in Amazake (Part1) determination on sugar composition. J. Jpn. Soy Sauce Res. Inst. 1977, 3, 105-110.

9. Oguro, Y.; Nishiwaki, T.; Shinada, R.; Kobayashi, K.; Kurahashi, A. Metabolite profile of koji amazake and its lactic acid fermentation product by Lactobacillus sakei UONUMA. J. Biosci. Bioeng. 2017, 124, 178-183. [CrossRef]

10. Oguro, Y.; Nakamura, A.; Kurahashi, A. Effect of temperature on saccharification and oligosaccharide production efficiency in koji amazake. J. Biosci. Bioeng. 2019, 127, 570-574. [CrossRef] [PubMed]

11. Tukiyama, R.; Maeda, T.; Miyashita, K.; Shikata, H.; Ishigami, Y. Components in Amazake (Part2) amino acids, organic acids composition, and other Composition. J. Jpn. Soy Sauce Res. Inst. 1977, 3, $205-209$.

12. Nagao, Y.; Sata, M. Effect of a late evening snack of Amazake in patients with liver cirrhosis: A pilot study. J. Nutr. Food Sci. 2013, 3, 1000223. [CrossRef]

13. Fukui, S.; Tani, Y.; Kishibe, T. Studies on the role of B-vitamins in Sake-brewing (VI): Change of the amounts of several B-vitamins in the Process of Koji-manufacture. J. Ferment. Technol. 1955, 33, 239-242.

14. Lee, D.E.; Lee, S.; Jang, E.S.; Shin, H.W.; Moon, B.S.; Lee, C.H. Metabolomic profiles of Aspergillus oryzae and Bacillus amyloliquefaciens during rice Koji fermentation. Molecules 2016, 21, 773. [CrossRef]

15. Tanabe, Y.; Maruyama, J.; Yamaoka, S.; Yahagi, D.; Matsuo, I.; Tsutsumi, N.; Kitamoto, K. Peroxisomes are involved in biotin biosynthesis in Aspergillus and Arabidopsis. J. Biol. Chem. 2011, 286, 30455-30461. [CrossRef] [PubMed]

16. Fujino, Y.; Ohnishi, M. Structure of cerebroside in Aspergillus oryzae. Biochim. Biophys. Acta 1976, 486, $161-171$.

17. Sakamoto, M.; Sakatani, M.; Ferdouse, J.; Hamajima, H.; Tusge, K.; Nishimnukai, M.; Yanagita, T.; Nagao, K.; Mitsutake, S.; Kitagaki, H. Development of a quantitative method for the contents of glycosylceramide contained in Japanese foods brewed with koji and its application. J. Brew. Soc. Jpn. 2017, 112, 655-662.

18. Hamajima, H.; Fujikawa, A.; Yamashiro, M.; Ogami, T.; Kitamura, S.; Tsubata, M.; Tan, S.; Matsunaga, H.; Sawada, K.; Kumagai, S.; et al. Chemical analysis of the sugar moiety of monohexosylceramide contained in Koji, Japanese traditional rice fermented with Aspergillus. Fermentation 2016, 2, 2. [CrossRef]

19. Saigusa, N.; Ohba, R. Effects of koji production and saccharification time on the antioxidant activity of amazake. Food Sci. Technol. Res. 2007, 13, 162-165. [CrossRef]

20. Watanabe, T. Ingredients in "Sake Cake" contribute to healthy and beauty. J. Brew. Soc. Jpn. 2012, 107, 282-291. [CrossRef]

21. Imanari, T.; Tamamura, Z. The identification of a-ethyl glucoside and sugar alcohol in sake. Agric. Biol. Chem. 1971, 35, 321-324. [CrossRef]

22. Kashimura, O.; Uehara, Y.; Shimazaki, A. Effects of amazake using rice koji intake in sports athletes on physical fatigue and subjective symptoms during exercise training. Jpn. J. Sport Health Sci. 2019, 6, 37-48.

23. Matsumoto, K.; Koba, T.; Hamada, K.; Sakurai, M.; Higuchi, T.; Miyata, H. Branched-chain amino acid supplementation attenuates muscle soreness, muscle damage and inflammation during an intensive training program. J. Sports Med. Phys. Fit. $2009,49,424-431$.

24. Sumiyoshi, K.; Nakao, M. Effect of Amazake ingestion on constipation. Jpn. J. Nurs. Art Sci. 2017, 16, 36-40.

25. Sakurai, M.; Kubota, M.; Iguchi, A.; Shigematsu, T.; Yamaguchi, T.; Nakagawa, S.; Kurahashi, A.; Oguro, Y.; Nishiwaki, T.; Aihara, K.; et al. Effects of Koji amazake and its lactic acid fermentation product by Lactobacillus sakei UONUMA on defecation status in healthy volunteers with relatively low stool frequency. Food Sci. Technol. Res. 2019, 25, 853-861. [CrossRef] 
26. Mori, S.; Tanaka, Y.; Watabe, K.; Tamada, M.; Morita, M.; Matsuo, T. Amazake using the lees and rice Koji promotes regular movements-A randomized, placebo-controlled parallel-group comparison study. Jpn. Pharmacol. Ther. 2019, 47, 759-765.

27. Mori, S.; Morita, M.; Matsuo, T. Effect of oral intake of Amazake containing Sake lee and rice Koji on the human intestinal microbiota of Amazake using-The randomized placebo-controlled crossover comparison study. Jpn. Pharmacol. Ther. 2020, 48, 1187-1193.

28. Maruki-Uchida, H.; Sai, M.; Yano, S.; Morita, M.; Maeda, K. Amazake made from sake cake and rice koji suppresses sebum content in differentiated hamster sebocytes and improves skin properties in humans. Biosci. Biotechnol. Biochem. 2020, 84, 1689-1695. [CrossRef]

29. Kawakami, S.; Ito, R.; Maruki-Uchida, H.; Kamei, A.; Yasuoka, A.; Toyoda, T.; Ishijima, T.; Nishimura, E.; Morita, M.; Sai, M.; et al. Intake of a mixture of Sake cake and rice malt increases mucin levels and changes in intestinal microbiota in mice. Nutrients 2020, 12, 449. [CrossRef]

30. Hamajima, H.; Matsunaga, H.; Fujikawa, A.; Sato, T.; Mitsutake, S.; Yanagita, T.; Nagao, K.; Nakayama, J.; Kitagaki, H. Japanese traditional dietary fungus koji Aspergillus oryzae functions as a prebiotic for Blautia coccoides through glycosylceramide: Japanese dietary fungus koji is a new prebiotic. SpringerPlus 2016, 5, 1321. [CrossRef] [PubMed]

31. Ueda, M.; Kitagawa, M.; Koike, S.; Yamamoto, T.; Kondo, S. Effect of intake of Amazake on skin barrier functions in healthy adult women subjects-A randomized, double-blind, placebo-controlled study. Jpn. Pharmacol. Ther. 2017, 45, 1811-1820.

32. Hirakawa, S.; Sato, A.; Hattori, Y.; Matsumoto, T.; Yokoyama, K.; Kanai, S. Dietary rice bran extract improves TEWL of whole body. Jpn. Pharmacol. Ther. 2013, 41, 1051-1059.

33. Asai, S.; Miyachi, H. Evaluation of skin-moisturizing effect of oral or percutaneous use of plant ceramides. Rinsho Byori 2007, 55, 209-217.

34. Uchiyama, T.; Nakano, Y.; Ueda, O.; Mori, H.; Nakashima, M.; Noda, A.; Ishizaki, C.; Mizoguchi, M. Oral intake of glucosylceramide improves relatively higher level of transepidermal water loss in mice and healthy human subjects. J. Health Sci. 2008, 54, 559-566. [CrossRef]

35. Hori, M.; Kishimoto, S.; Tezuka, Y.; Nishigori, H.; Nomoto, K.; Hamada, U.; Yonei, Y. Double-blind study on effects of glucosyl ceramide in beet extract on skin elasticity and fibronectin production in human dermal fibroblasts. Anti Aging Med. 2010, 7 , 129-142. [CrossRef]

36. Shibata, K.; Tsubouchi, R. Clinical effects of N-acetylglucosamine supplementation on dry skin. Aesthetic Dermatol. 2008, 18, 91-99.

37. Kurahashi, A.; Nakamura, A.; Oguro, Y.; Yonei, Y. Safety evaluation of a long-term intake of koji amazake. J. Brew. Soc. Jpn. 2020, $115,159-172$.

38. Hseu, Y.C.; Vudhya Gowrisankar, Y.; Chen, X.Z.; Yang, Y.C.; Yang, H.L. The antiaging activity of ergothioneine in UVA-irradiated human dermal fibroblasts via the inhibition of the AP-1 pathway and the activation of Nrf2-mediated antioxidant genes. Oxid. Med. Cell Longev. 2020, 2020, 2576823. [CrossRef]

39. Kitamura, N.; Ota, Y.; Haratake, A.; Ikemoto, T.; Tanno, O.; Horikoshi, T. Effects of ethyl $\alpha$-D-glucoside on skin barrier disruption. Skin Pharmacol. Physiol. 1997, 10, 153-159. [CrossRef]

40. Bogaki, T.; Mitani, K.; Oura, Y.; Ozeki, K. Effects of ethyl- $\alpha$-D-glucoside on human dermal fibroblasts. Biosci. Biotechnol. Biochem. 2017, 81, 1706-1711. [CrossRef]

41. Kubomura, D.; Ueno, T.; Yamada, M.; Tomonaga, A.; Nagaoka, I. Effect of N-acetylglucosamine administration on cartilage metabolism and safety in healthy subjects without symptoms of arthritis: A case report. Exp. Ther. Med. 2017, 13, 1614-1621. [CrossRef]

42. Kurahashi, A.; Nakamura, A.; Oguro, Y.; Watanabe, K.; Ozaki, N.; Goto, H.; Hirayama, M. Beneficial effects of koji amazake in suppressing the postpradial increase in blood glucose and insulin levels in healthy adults. J. Brew. Soc. Jpn. 2020, 115, 43-53.

43. Ishida, Y.; Sadakiyo, T.; Inoue, S.; Watanabe, H.; Mitsuzumi, H.; Fukuda, S.; Ushio, S.; Hiramatsu, J. The attenuating effect of isomaltodextrin on postprandial blood glucose level in healthy human subjects-A randomized, placebo-controlled, double-blind crossover study. Jpn. Pharmacol. Ther. 2017, 45, 1179-1185.

44. Kashimura, J.; Nagai, Y. Inhibitory effect of palatinose on glucose absorption in everted rat gut. J. Nutr. Sci. Vitaminol. 2007, 53, 87-89. [CrossRef]

45. Hamajima, H.; Tanaka, M.; Miyagawa, M.; Sakamoto, M.; Nakamura, T.; Yanagita, T.; Nishimukai, M.; Mitsutake, S.; Nakayama, J.; Nagao, K.; et al. Koji glycosylceramide commonly contained in Japanese traditional fermented foods alters cholesterol metabolism in obese mice. Biosci. Biotechnol. Biochem. 2019, 83, 1514-1522. [CrossRef]

46. Oura, S.; Suzuki, S.; Hata, Y.; Kawato, A.; Abe, Y. Evaluation of physiological functionalities of amazake in mice. J. Brew. Soc. Jpn. 2007, 102, 781-788. [CrossRef]

47. WHO. Guideline: Sugars Intake for Adults and Children. 2015. Available online: https://www.who.int/publications/i/item/97 89241549028 (accessed on 15 May 2021).

48. Kurahashi, A.; Yonei, Y. Effects and safety of koji amazake: An excessive intake test. J. Brew. Soc. Jpn. 2019, 114, 654-662.

49. Deguchi, Y.; Osada, K.; Uchida, K.; Kimura, H.; Yoshikawa, M.; Kudo, T.; Yasui, H.; Watanuki, M. Effects of Extract of guava leaves on the development of diabetes in the $\mathrm{db} / \mathrm{db}$ mouse and on the postprandial blood glucose of human subjects. Nippon Nogeikagaku Kaishi 1998, 72, 923-931. [CrossRef]

50. Hayashi, N.; Oga, H.; Kishimoto, Y.; Tagami, H. Effects of resistant hydrogenated starch hydrolysate on postprandial blood glucose levels. J. Jpn. Nutr. Food Sci. 2006, 59, 247-253. [CrossRef] 
51. Ui, S. Fundamental Studies on the Food Poisoning by Amasake. I. Epidemiological Investigation on the Amasake Food Poisoning. Jpn. J. Hyg. 1959, 14, 707-712. [CrossRef]

52. Ui, S. Fundamental Studies on the Food Poisoning by Amasake. II. Food-sanitation on Amasake. Jpn. J. Hyg. 1959, 14, 713-716. [CrossRef]

53. Ui, S. Fundamental Studies on the Food Poisoning by Amasake. III. Growth of Bacteria in Amasake. Jpn. J. Hyg. 1959, 14, 717-723. [CrossRef] 